\title{
Sirenomelia, the Fetal 'Mermaid': Ultrasound and Magnetic Resonance Features
}

\author{
Navneet Sharma, Neeraj Kumar, Anupam Jhobta and Rohit Bhoil ${ }^{*}$ \\ Department of Radiology, IGMC Shimla (HP), India
}

\begin{abstract}
Sirenomelia (otherwise known as Mermaid Syndrome), is a very rare congenital anomaly characterized by variable fusion of lower limbs along with thoracolumbar spinal anomalies, sacrococcygeal agenesis, genitourinary and gastrointestinal tract malformations, with an incidence of 0.8 to 1 case per 100,000 births. This anomaly has a strong association with maternal diabetes, and some researchers consider it to be a severe form of caudal regression syndrome. It is a lethal anomaly, with half of the cases being stillborn and most cases involving live births dying within a few days. We present here a radiological evaluation of a case of sirenomelia in a 28-year-old primigravida with history of occasional cocaine and alcohol use. The fetus had duodenal atrasia, bilateral renal agenesis, caudal dysgenesis, imperforate anus and absent genitalia, as determined by fetal sonogram and fetal magnetic resonance imaging (MRI), which had been carried out for better evaluation. Fetal sonogram revealed sacral dysgenesis, and double bubble sign suggestive of duodenal atresia. Fetal MRI showed bilateral renal agenesis, duodenal atresia and single hypoplastic lower limb. One of two upper limbs was also found to be hypoplastic. After delivery, the stillborn was examined externally. There were no external genitalia and the anus was imperforate. There was a single hypoplastic lower limb. One of two upper limbs was also hypoplastic. Early diagnosis of sirenomelia is necessary for safe termination of pregnancy. However, complete evaluation of a sirenomelia fetus is hindered by associated severe oligohydramnios. Fetal MRI has no such constraints and can demonstrate various anomalies in a greater detail than fetal sonogram.
\end{abstract}

Introduction

Sirenomelia or Mermaid Syndrome is a rare, congenital and lethal syndrome characterized by variable fusion of lower limbs and associated with array of visceral anomalies. Incidence of this syndrome is $0.8-1 / 100,000$ births. ${ }^{1}$ Approximately 300 cases have been reported so far in the worldwide literature, including 10 cases from India. ${ }^{2}$ The etiopathogenesis of this disease is not clear, however a strong association with maternal diabetes has been noted. ${ }^{2,3}$ Here, we present radiological evaluation of a case of sirenomelia with associated duodenal atresia, bilateral renal agenesis, caudal dysgenesis, imperforate anus and absent genitalia; the deformity was diagnosed on fetal sonogram and fetal magnetic resonance imaging (MRI), which had been performed for better evaluation of the congenital malformations. Written informed consent was obtained from the mother to publish this

Keywords: Sirenomelia; Magnetic resonance imaging, MRI; Mermaid Syndrome. Abbreviations: MRI, magnetic resonance and imaging.

Received: December 01, 2016; Revised: June 21, 2017; Accepted: July 19, 2017

${ }^{*}$ Correspondence to: Rohit Bhoil, Department of Radiology, IGMC, Shimla (HP) 171001, India. Tel: +91-9418021905, E-mail: rohitbhoil@gmail.com

How to cite this article: Sharma N, Kumar N, Jhobta A, Bhoil R. Sirenomelia, the Fetal 'Mermaid': Ultrasound and Magnetic Resonance Features. Exploratory Research and Hypothesis in Medicine 2017;2(3):68-71. doi: 10.14218/ERHM.2016.00026. case report.

Case report

A 28-year-old primigravida presented for antenatal sonogram at 26 weeks of gestation. History-taking revealed occasional cocaine and alcohol use but no symptoms or diagnosis of diabetes mellitus. No other significant personal or family history was reported. No karyotyping was performed.

Fetal sonogram (Fig. 1) revealed a single intrauterine fetus with normal cardiac activity. However, there was caudal dysgenesis, as sacral vertebrae were not visualized. Double bubble sign suggestive of duodenal atresia was also seen. In addition, both kidneys and lower limbs were not visualized. There was only a single umbilical artery. The fetal sonogram was suboptimal due to severe oligohydramnios.

Antenatal fetal MRI (Fig. 2) was carried out for better visualization of the detected congenital malformations. The ultrasound findings of bilateral renal agenesis and duodenal atresia detected were confirmed by MRI. The MRI also showed presence of a single hypoplastic lower limb, as well as a hypoplastic upper limb (the other of the two appearing normal).

Based on the findings from antenatal sonogram and antenatal MRI, the diagnosis of "sirenomelia (sympus apus)" was made. The 

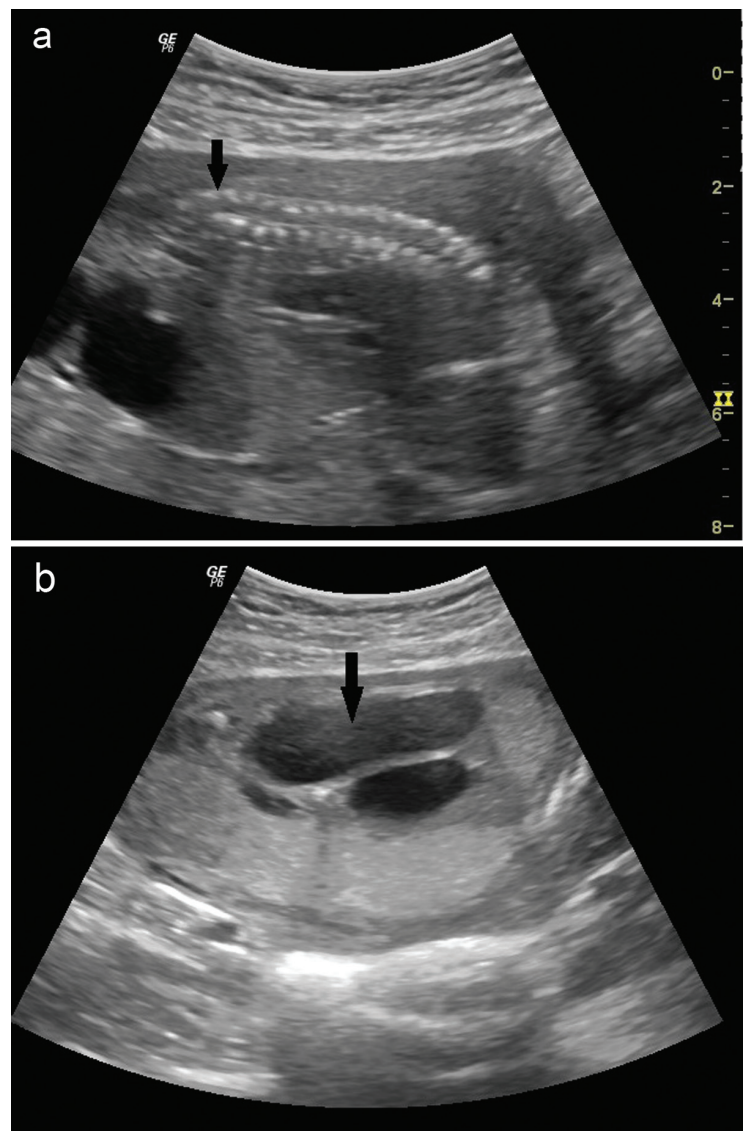

Fig. 1. A 28-year-old female at 26 weeks of gestation 26 . Ultrasound images showing sacral agenesis (a, arrow) and double bubble sign ( $b$, arrow) suggestive of duodenal atresia, with severe oligohydramnios.

pregnancy was terminated at 28 weeks of gestation by induction of labor. A stillborn child (Fig. 3) was delivered, weighing 1160 grams. The placenta was small, thin and irregular, and weighed $350 \mathrm{gm}$. On physical examination, external genitalia were absent and there was imperforate anus. There was a single hypoplastic lower limb which was inverted and externally rotated with the sole facing anteriorly. A hypoplastic right upper limb was also noted. However, neither X-ray nor autopsy were performed, as the parents did not give consent.

\section{Discussion}

Sirenomelia or Mermaid Syndrome, is a very rare congenital deformity characterized by variable fusion of lower limbs along with thoracolumbar spinal anomalies, sacrococcygeal agenesis, genitourinary and gastrointestinal tract malformations..$^{2-5}$ Due to resemblance of these deformed fetuses to the mermaid (Siren) of Greek mythology, it was given the common name of Mermaid Syndrome. The incidence of sirenomelia is $0.8-1$ case $/ 100,000$ births, with a male to female ratio of $3: 1,{ }^{1,2}$ While many theories have been proposed for the cause of sirenomelia, none is considered conclusive. There is a strong association with maternal diabetes, as $22 \%$ of cases have a diabetic mother. ${ }^{4-5}$ The condition is 100 times more likely to occur in identical twins than in single births or dizygotic twins. ${ }^{4}$ Fusion of the lower extremities, presence of single umbilical and
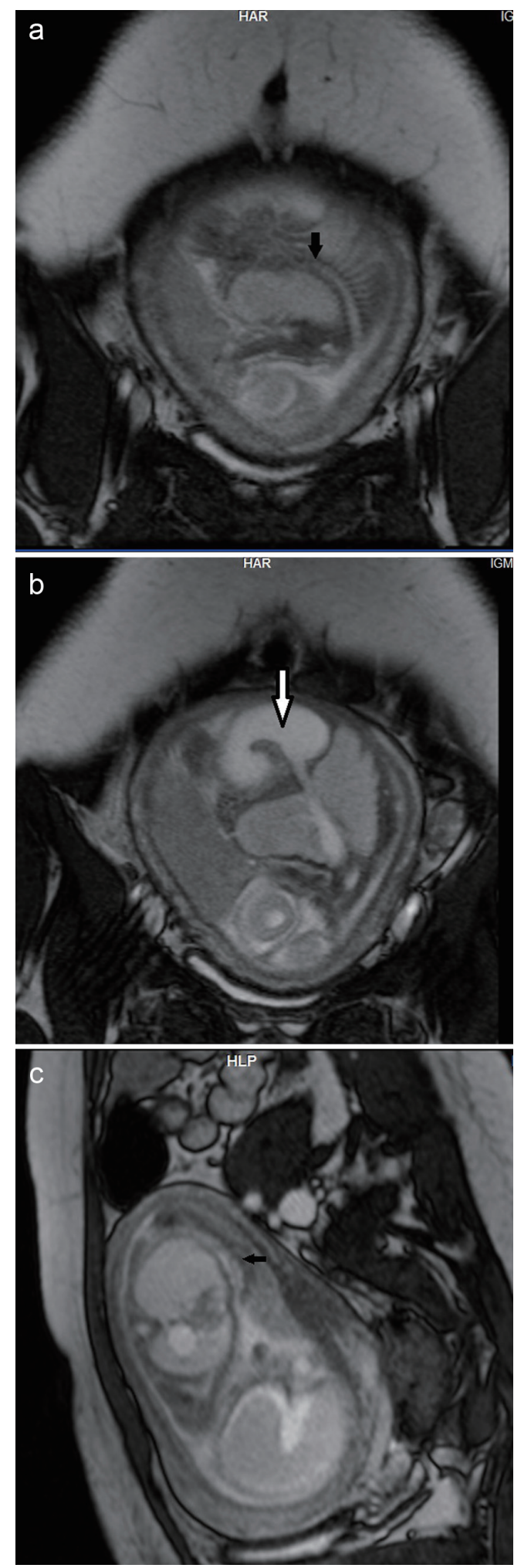

Fig. 2. Magnetic resonance images of the same patient. Coronal halfFourier-acquired single-shot turbo spin echo (HASTE) image showing sacral agenesis ( $a$, arrow) and duodenal atresia ( $b$, arrow) with non-visualized bilateral kidneys. True fast-imaging with steady-state precession (FISP) showing a single lower limb (c, arrow head) and a hypoplastic upper limb. 


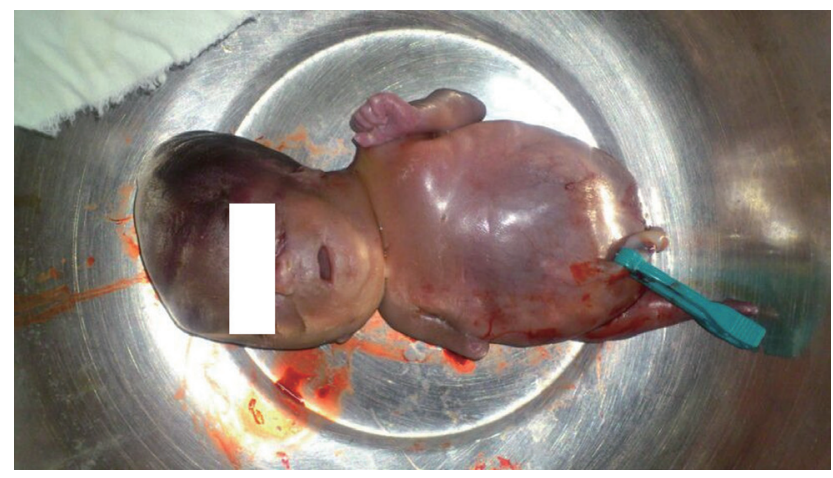

Fig. 3. Image of stillborn showing a single hypoplastic lower limb, hypoplastic right upper limb and absent external genitalia.

persistent vitelline artery are major features of sirenomelia. ${ }^{3-6}$

Hibelink et al. ${ }^{7}$ demonstrated that administration of cadmium and lead can produce sirenomelia in the golden hamster. Similarly, the possible teratogenic effects of Vitamin A have been proposed in the etiology of this condition. ${ }^{7}$ Some researchers consider this anomaly as a manifestation of the caudal regression syndrome, which is a consequence of abnormal development of structures derived from the caudal mesoderm of the embryo. ${ }^{8-10}$ Maternal diabetes mellitus, genetic predisposition and vascular hypoperfusion have been proposed as possible causative factors. ${ }^{7-11}$

Lately, many authors have drawn attention to the overlap in phenotypic manifestations of sirenomelia and the so-called VACTERL anomalies (vertebral defects, anal atresia, cardiac defects, tracheo-esophageal fistula, renal anomalies, and limb abnormalities). ${ }^{9}$ In 1987, Stocker and Heifetz proposed the defect in the primitive streak as a causative factor. ${ }^{12}$ Stevenson et al. ${ }^{13}$ proposed the vitelline artery steal theory. They reported that a large artery arose from the aorta, high in the abdomen and beyond which the aorta and its branches were hypoplastic in each of the 11 sirenomelia specimens; the conclusion was that the vitelline artery diverts/ steals blood and nutrients away from the caudal part of the embryo.

Various anomalies present in sirenomelia include varying degree of fusion (symelia) and/or hypoplasia of lower limbs, vertebral anomalies (sacral ageneis), anorectal agenesis, urinary tract anomalies (bilateral renal agenesis; ureteral, vesical and urethral agenesis) and genital anomalies (Wolffian or Mullerian duct agenesis). ${ }^{7-11}$ Sirenomelia is fatal, and about $50 \%$ of cases are stillborn because of bilateral renal agenesis and associated visceral anomalies. ${ }^{10,11}$ Anomalies of kidneys, significant oligohydramnios, and associated hypoplasia of the lungs (Potter's syndrome) make a very unfavorable prognosis for sirenomelia. ${ }^{9-11}$ Many researchers have considered sirenomelia to be a severe form of caudal regression syndrome because of the observed abnormal development of fetal caudal mesodermal structures before the $4^{\text {th }}$ week of gestation, that later extends to different craniocaudal levels. It may also lead to the absence of genitalia and renal agenesis if paramesonephric and mesonephric ducts are involved. Survival depends upon visceral anomalies instead of sirenomelia. ${ }^{7-12}$

The syndrome is classified into three types according to the number of feet: simpus apus, no feet present; simpus unipus, one foot; simpus dipus, two feet. ${ }^{13-17}$ Our case was of the simpus apus variety.

\section{Conclusions}

Since sirenomelia is almost always fatal, early antenatal diagnosis is necessary for prenatal counseling for possible safe pregnancy termination. However, complete evaluation of a sirenomelia fetus is hindered by associated severe oligohydramnios. Fetal MRI has no such constraints and can demonstrate various anomalies in greater detail than fetal sonogram, especially in late (after 20 weeks) gestation.

\section{Conflict of interest}

The authors have no conflict of interests related to this publication.

\section{Author contributions}

Data collection (NS), data interpretation (NS, NK), data ananlysis (NK, AJ), manuscript writing (NK, RB), supervision (AJ), manuscript revising $(\mathrm{RB})$, case preparation $(\mathrm{RB})$.

\section{References}

[1] Dharmraj M, Gaur S. Sirenomelia: a rare case of foetal congenital anomaly. J Clin Neonatol 2012;1(4):221-223. doi:10.4103/2249-4847. 106006.

[2] Sahu L, Singh S, Gandhi G, Agarwal K. Sirenomelia: a case report with literature review. Int J Reprod Contracept Obstet Gynecol 2013;2(3):430-432. doi:10.5455/2320-1770.ijrcog20130936.

[3] Reddy KR, Srinivas S, Kumar S, Reddy S, Hariprasad, Irfan GM. Sirenomelia a rare presentation. J Neonatal Surg 2012;1(1):7.

[4] Reviews CT. e-study guide for: langmans medical embryology. Cram101 Textbook Reviews 2012:295.

[5] Duhamel B. From the mermaid to anal imperforation: the syndrome of caudal regression. Arch Dis Child 1961;36(186):152-155.

[6] Naveena S, Mrudula C. Sirenomelia - the mermaid syndrome: a case report. IOSR J Dent Med Sci 2013;7:1-4.

[7] Hilbelink DR, Kaplan S. Sirenomelia: analysis in the cadmiumand lead-treated golden hamster. Teratog Carcinog Mutagen 1986;6(5):431-440.

[8] Das SP, Ojha N, Ganesh GS, Mohanty RN. Conjoined legs: sirenomelia or caudal regression syndrome? Indian J Orthop 2013;47(4):413416. doi:10.4103/0019-5413.114936.

[9] Das BB, Rajegowda BK, Bainbridge R, Giampietro PF. Caudal regression syndrome versus sirenomelia: a case report. J Perinatol 2002;Mar22(2):168-170. doi:10.1038/sj.jp.7210598.

[10] Assimakopoulos E, Athanasiadis A, Zafrakas M, Dragoumis K, Bontis J. Caudal regression syndrome and sirenomelia in only one twin in two diabetic pregnancies. Clin Exp Obstet Gynecol 2004;31(2):151-153.

[11] Barr M Jr. Comments on "origin of abnormality in a human simelian foetus as elucidated by our knowledge of vertebrate development". Teratology 1988;38(5):487-491. doi:10.1002/tera.1420380513.

[12] Garrido-Allepuz C, Haro E, González-Lamuño D, Martínez-Frías ML, Bertocchini F, Ros MA. A clinical and experimental overview of sirenomelia: insight into the mechanisms of congenital limb malformations. Dis Model Mech 2011;4(3):289-299. doi:10.1242/ dmm.007732.

[13] Stevenson RE, Jones KL, Phelan MC, Jones MC, Barr M Jr, Clericuzio $C$, et al. Vascular steal: the pathogenetic mechanism producing sirenomelia and associated defects of the viscera and soft tissues. Pediatrics 1986;78:451-457.

[14] Sadler TW, Rasmussen SA. Examining the evidence for vascular pathogenesis of selected birth defects. Am J Med Genet A 2010;152A(10):2426-2436. doi:10.1002/ajmg.a.33636.

[15] Duesterhoeft SM, Ernst LM, Siebert JR, Kapur RP. Five cases of caudal regression with an aberrant abdominal umbilical artery: further support for a caudal regression-sirenomelia spectrum. Am J Med Genet A 2007;143A(24):3175-3184. doi:10.1002/ajmg.a.32028. 
[16] KB Taori, K Mitra, NP Ghonga, RO Gandhi, T Mammen, J Sahu. Sirenomelia sequence (mermaid): report of three cases. Indian J Radiol Imaging 2002;12(3):399-401.
[17] Samal SK, Rathod S. Sirenomelia: The mermaid syndrome: report of two cases. J Nat Sci Biol Med 2015;6(1):264-266. doi:10.4103/09769668.149227 